\title{
DECOMPOSITIONS OF MODULES OVER A DISCRETE VALUATION RING
}

\author{
ROBERT O. STANTON
}

(Received 14 March 1978)

Communicated by $\mathbf{R}$. Lidl

\begin{abstract}
Let $N$ be a direct summand of a module which is a direct sum of modules of torsion-free rank one over a discrete valuation ring. Then there is a torsion module $T$ such that $N \oplus T$ is also a direct sum of modules of torsion-free rank one.
\end{abstract}

Subject classification (Amer. Maths. Soc. (MOS) 1970): primary 13 C 05; secondary 13 H 99, $20 \mathrm{~K} 25,18 \mathrm{E} 15$.

\section{Introduction}

$R$ will denote a fixed discrete valuation ring, and module will mean $R$-module. The generator of the unique maximal ideal of $R$ will be denoted $p$. The quotient ring of $R$ will be represented by $Q$. If $X$ is a subset of the module $M$, then $[X]$ represents the submodule generated by $X . E(M)$ will denote the endomorphism ring of the module $M$. A module $M$ has torsion-free rank one if, given elements $m$ and $n$ of infinite order in $M$, there are nonzero elements $r$ and $s$ in $R$ such that $r m=s n$. A module is completely decomposable if it is a direct sum of modules of torsion-free rank one. Two modules $M$ and $N$ are almost isomorphic if there are torsion modules $S$ and $T$ such that $M \oplus S$ is isomorphic to $N \oplus T$. We may now state the main theorem of the paper.

THEOREM 1. Let $N$ be a direct summand of a completely decomposable module $M=\bigoplus_{I} M_{i}$, where each $M_{i}$ has torsion-free rank one. Then there is a subset $J$ of $I$ such that $N$ is almost isomorphic to $\bigoplus_{J} M_{i}$.

A lengthy direct proof of this theorem appeared in the author's doctoral dissertation (Stanton (1973)), which was written under the direction of Professor 
Elbert Walker. Categorical methods developed by C. Walker and Warfield (1976), along with a category defined by $\mathrm{E}$. Walker, have resulted in substantial simplifications. The latter approach will be followed here. The special case of Theorem 1 in which $M$ is a simply presented module is a consequence of the following result of Warfield.

TheOREM 2. (Warfield (1975), Theorem 5.2.) Let $M$ and $N$ be summands of simply presented modules. Then $M \simeq N$ if and only if $f(\alpha, M)=f(\alpha, N)$ for all ordinals $\alpha$ and for $\alpha=\infty$, and $S(e, M)=S(e, N)$ for all equivalence classes $e$ of indicators.

In the above theorem, $f$ represents the UIm invariants while $S$ represents the invariants in Stanton (1975).

In the next section, a proof of Theorem 1 is given and some consequences of the theorem are discussed. Lemmas 5 and 6, as well as Corollaries 7,9 and 10, originally appeared in the author's dissertation (Stanton (1973)).

\section{The proof of Theorem 1}

In the following discussion, we will be working in an additive category (see MacLane (1967), pp. 249-253). The three definitions below, as well as Theorem 3, are due to C. Walker and Warfield (1976).

DefinITION. An additive category satisfies a weak Grothendieck condition if for every index set $I$ and every nonzero monic $A \rightarrow \bigoplus_{I} B_{i}$, there is a finite subset $J$ of $I$ and a commutative diagram

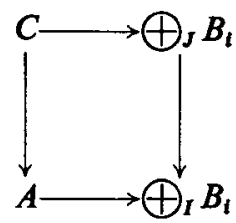

with the map $C \rightarrow A$ nonzero.

Definition. An object $S$ in an additive category is small if every map $f: S \rightarrow \bigoplus_{\mathrm{r}} M_{\mathrm{t}}$ factors through a subsum $\bigoplus_{F} M_{i}$, for some finite subset $F$ of $I$.

Definition. An object $M$ is countably finitely approximable if there is a countable family $\left\{f_{i}: S_{l} \rightarrow M\right\}_{i=1}^{\infty}$ of maps with each $S_{l}$ small, with the property that a monic $f: L \rightarrow M$ is an isomorphism if and only if each $f_{l}$ can be factored through $f$.

Note that a small object is trivially countably finitely approximable.

Theorem. 3. (C. Walker, Warfield (1976), Theorem 5.) Let $\mathscr{C}$ be an additive category with kernels and infinite sums which satisfies a weak Grothendieck condition. 
If $M=\oplus_{I} M_{i}=N \oplus K$, with each $M_{i}$ countably finitely approximable, and with the endomorphism ring of each $M_{\ell}$ a local ring, then $N$ is isomorphic to a direct sum $\bigoplus_{J} M_{i}$, for some subset $J$ of $I$. Consequently, any two direct decompositions of $M$ have isomorphic refinements.

We now define a category which will allow the use of Theorem 3 to prove Theorem 1 . Let $\mathscr{C}$ be the category (defined by $\mathrm{E}$. Walker) whose objects are modules, and whose morphisms are given by

$$
\operatorname{Hom}(M, N)=\operatorname{Hom}_{R}(M, N) / I(M, N)
$$

where $A$ is a class of modules closed under submodules, homomorphic images and direct sums, and

$$
I(M, N)=\left\{\varphi \in \operatorname{Hom}_{R}(M, N): \varphi(M) \in A\right\} .
$$

$\mathscr{C}$ is easily seen to be an additive category with a weak Grothendieck condition. Any object in $A$ is equivalent to 0 in $\mathscr{C}$. In the sequel, the notation $\operatorname{Hom}(M, N)$ will always refer to the group of morphisms of this category. $\operatorname{Hom}(M, M)$ may be considered as a ring in the usual way.

THEOREM 4. (E. Walker.) Let $M$ and $N$ be modules. Then $M$ and $N$ are equivalent as objects of $\mathscr{C}$ if and only if there are modules $S$ and $T$ in $A$ such that $M \oplus S \simeq N \oplus T$ as modules.

Proof. The latter condition clearly implies the former. Suppose that $M$ and $N$ are equivalent. Then there are homomorphisms $\alpha: M \rightarrow N, \beta: N \rightarrow M, \rho: M \rightarrow M$ and $\sigma: N \rightarrow N$ such that

$$
\beta \alpha=1_{M}+\rho, \quad \alpha \beta=1_{N}+\sigma
$$

and such that the image $T$ of $\rho$ and the image $S$ of $\sigma$ are in $A$. We claim that $M \oplus S=N \oplus T$. Let $\zeta: M \oplus S \rightarrow N \oplus T$ and $\xi: N \oplus T \rightarrow M \oplus S$ be defined by

$$
\begin{gathered}
\zeta(m, s)=(\alpha m-s, \rho m-\beta s), \\
\xi(n, t)=(\beta n-t, \sigma n-\alpha t) .
\end{gathered}
$$

The identities $\alpha \rho=\sigma \alpha$ and $\beta \sigma=\rho \beta$ can be used to show the ranges of $\zeta$ and $\xi$ are as claimed. Routine calculations show that $\zeta$ and $\xi$ are inverses of each other.

LEMMA 5. Let $M$ be a module of torsion-free rank one such that the divisible part of $M$ is a torsion module and let $\varphi \in E(M)$. Then there is an element $m$ of infinite order in $M$ such that $\varphi$ restricted to $[m]$ is multiplication by an element of $R$.

Proof. Pick $x$ of infinite order in $M$ and let $\varphi(x)=y$. If $y$ is a torsion element, then $\varphi\left(p^{k} x\right)=0$ for some $k \geqslant 0$, so we may select $m$ to be $p^{k} x$. If $y$ is not torsion, there is a unit $a$ of $R$, and $j, k \geqslant 0$ such that $p^{k} a x=p^{j} y$. Then $\varphi\left(p^{k} a x\right)=\varphi\left(p^{j} y\right)=$ 
$p^{k} a y$, and a comparison of heights guarantees that $k \geqslant j$. Letting $m=p^{j} a x, \varphi$ restricted to $[m]$ is multiplication by $p^{k-J} a$.

Henceforth, the class $A$ in the definition of Walker's category $\mathscr{C}$ will consist of the class of all torsion modules.

Lemma 6. Let $M$ be a module of torsion-free rank one such that the divisible part of $M$ is a torsion module. Considered as an $R$-module, $E(M)=R \oplus S$, where $R$ represents multiplication by elements of $R$, and $S=I(M, M)$.

Proof. It is easily seen that $S$ is an $R$-module and that $R \cap S=0$. If $\varphi \in E(M)$, then there is $r \in R$ and $m \in M$ such that $m$ has infinite order and $\varphi(m)=r m$, by Lemma 5 . So $\varphi=r+(\varphi-r)$, where the first term is in $R$ and the second is in $S$.

COROLlary 7. If $M$ is a module of torsion-free rank one, then $\operatorname{Hom}(M, M)$, its endomorphism ring in $\mathscr{C}$, is local.

Proof. If the divisible part of $M$ is torsion, this follows from Lemma 6. Otherwise $M=Q \oplus T$, where $T$ is torsion, and again $\operatorname{Hom}(M, M)$ is local.

LEMMA 8. If $M$ is a module of torsion-free rank one, then $M$ is small as an object of $\mathscr{C}$.

Proof. Let $\varphi: M \rightarrow \bigoplus_{I} M_{l}$ be a homomorphism, and let $\pi_{i}: \bigoplus_{I} M_{i} \rightarrow M_{i}$ be the projection for each $i$. Select an element $m \in M$ of infinite order, and let $J$ be a subset of $I$ such that $I \backslash J$ is finite and $\pi_{i} \varphi m=0$ for all $i \in J$. Then for all $i \in J, \pi_{i} \varphi M$ is torsion, and thus equivalent to 0 in $\mathscr{C}$. Hence $M$ is small.

The proof of Theorem 1 can now be completed by observing that the hypotheses of Theorem 3 are satisfied and that equivalence in the category $\mathscr{C}$ is given by almost isomorphism by Theorem 4 .

We now give some consequences of Theorem 1. The definition of 'nice' is found in Fuchs (1973), p. 73, while 'decomposition basis' is defined in Rotman and Yen (1961), p. 251.

COROLlaRY 9. Let $N$ be a direct summand of a completely decomposable module $M=\bigoplus_{I} M_{i}$, where each $M_{i}$ has torsion-free rank one. Then

(i) $N$ has a nice decomposition basis.

(ii) There is a subset $J$ of $I$ such that $S(e, N)=S\left(e, \oplus_{J} M_{i}\right)$, for every equivalence class $e$ of indicators.

Proof. By Theorem 1, $N \oplus T_{1}=\left(\oplus_{J} M_{i}\right) \oplus T_{2}$, for some subset $J$ of $I$ and torsion modules $T_{1}$ and $T_{2}$. If $x_{i}$ is an element of infinite order in $M_{i}$, then $X=\left\{x_{i}: i \in J\right\}$ is a decomposition basis for $\left(\bigoplus, M_{i}\right) \oplus T_{2}$. For each $i \in J$, there is a nonzero 
element $r_{i} \in R$ such that $r_{i} x_{i} \in N$. Then $\left\{r_{l} y_{i}: i \in J\right\}$ is a decomposition basis for $N$, and is nice by Wallace (1971), Lemma 1, proving (i). The proof of (ii) follows from the fact that the $S$-invariants of torsion modules are all 0 .

E. Walker (1974) has pointed out that a significant simplification of the proof of Warfield's result (Theorem 2) is possible by using Corollary 9. The following result is a consequence of Theorem 2 .

COROLlary 10. Let $M$ and $N$ be direct summands of simply presented modules. Then $M$ is almost-isomorphic to $N$ if, and only if, for each equivalence class $e$ of indicators, $S(e, M)=S(e, N)$.

Proof. Let $S(e, M)=S(e, N)$. Then there are torsion modules $T_{1}$ and $T_{2}$ such that the Ulm invariants of $M \oplus T_{1}$ and $M \oplus T_{2}$ are equal. By Theorem 2, $M \oplus T_{1} \simeq$ $M \oplus T_{2}$.

\section{References}

L. Fuchs (1973), Infinite Abelian groups, Vol. II (Academic Press, New York).

S. MacLane (1967), Homology (Springer-Verlag, New York).

J. Rotman and T. Yen (1961), 'Modules over a complete discrete valuation ring', Trans. Amer. Math. Soc. 98, 242-254.

R. O. Stanton (1973), The A-exchange property applied to decompositions of modules over a discrete valuation ring (doctoral dissertation, New Mexico State University, Las Cruces).

R. O. Stanton (1975), 'An invariant for modules over a discrete valuation ring', Proc. Amer. Math. Soc. 49, 51-54.

C. L. Walker and R. B. Warfield, Jr. (1976), 'Unique decomposition and isomorphic refinement theorems in additive categories', J. Pure Appl. Algebra. 7 347-359.

E. A. Walker (1974), 'Remarks on T*-modules' (abstract), Notices Amer. Math. Soc. 21, A99.

K. D. Wallace (1971), 'On mixed groups of torsion free rank one with totally projective primary components', J. Algebra 17, 482-488.

R. B. Warfield, Jr. (1975), 'Classification theory of Abelian groups II, Local theory', to appear.

Department of Mathematics and Computer Science

St John's University

Jamaica, N.Y. 11439

USA 